\title{
Rheology, granule growth and granule strength: Application to the wet granulation of lactose-MCC mixtures
}

\author{
T.M. Chitu ${ }^{\text {a,* }}$, D. Oulahna ${ }^{a}$, M. Hemati ${ }^{b}$ \\ a RAPSODEE Research Centre, EMAC-CNRS FRE 3213, Ecole des Mines d'Albi-Carmaux, Campus Jarlard, 81013 Albi, France \\ ${ }^{\mathrm{b}}$ Laboratoires de Génie Chimique UMR-CNRS 5503, ENSIACET, INPT, BP 1301, 5 Rue Paulin Talabot, 31106 Toulouse Cedex 1, France \\ Corresponding author at Rapsodee IMT Mines-Albi (oulahna@mines-albi.fr)
}

Keywords:

Wet granulation

Rheology

Granule strength

Microcrystalline cellulose

Lactose

\section{A B S T R A C T}

This study aims at better understanding the wet granulation process of a binary mixture composed of microcrystalline cellulose (water insoluble) and lactose (water soluble). It investigates the effect of formulation (proportion of the different components in the mixture) on the granule growth kinetics, the evolution of granule morphology during granulation, the wet mass consistency and dry granule strength of the end product. Additionally the influence of mixer design has been studied by up scaling the process from the 1.9 L Mi-pro high shear mixer used as the reference scale to a $6 \mathrm{~L}$ Diosna P1-6 high shear mixer. The scale-up rules investigated were constant impeller tip speed and constant Froude number. Our results allowed us to draw the following conclusions:

The increase in MCC content is found to increase the optimum binder requirement for granulation, wet mass consistency and dry granule strength.

Granule growth takes place in three distinct stages: wetting, nucleation and growth. These stages can be identified with the help of the recorded torque values during the granulation process or by the evolution of granule size and granule morphology.

- The characterization of the starting materials by moisture sorption isotherms brings more insight to the role of each component during the granulation process.

The increase of the granulation scale has little influence on the observed growth mechanism. However bimodality of the granule size distribution is increased, wet mass consistency and dry granule strength are decreased with increasing scale of operation.

\section{Introduction}

The manufacturing of granules often demands the granulation of powder mixtures in order to obtain specific product properties at the end of the granulation process. Favoring one component over the other in binary mixtures influences granule properties. Microcrystalline cellulose (MCC) is one of the most widely used pelletization agents, being highly hygroscopic and conferring a certain degree of plasticity to the mixture while lactose is commonly used as excipient in the pharmaceutical industry. The amount of granulation liquid required for granulation depends on the mass fraction of MCC in the formulation as shown by Kristensen et al. [1] when describing the wet granulation of lactose-MCC mixtures in a rotary processor controlled by torque measurements. They show that increasing MCC content (between 10 and 100\%) increases the water content at the end-point of binder addition, determined as a function of torque evolution.
Kristensen et al. [2] compared the growth mechanisms of water soluble lactose with that of water insoluble calcium hydrogen phosphate. They found that calcium hydrogen phosphate requires 1.8 times more binder for significant granule growth than lactose. This observation was related to the increased plasticity of the wetted mass given by the solubility (about 20\%) of lactose in water.

Different authors (Kristensen et al. [1], Vecchio et al. [3] and Holm et al. [4]) recommend 10 to $45 \%$ mass percentage MCC for successful pelletization. However this amount depends largely on the other components present in the formulation as well as the grades of the components. Holm et al. [4] found that MCC content was influential when paired with the water soluble lactose than with the insoluble calcium hydrogen phosphate. They also found that the lowest level of MCC content for which a controllable pelletization process could be obtained increased with the fineness lactose and calcium phosphate grades. They also underlined the greater sensitivity of lactose-MCC formulations to binder content: within $0.25 \%$ relative to dry material translating to $\pm 15 \mathrm{~g}$ of water in order to achieve a mean pellet size of 900 to $1100 \mu \mathrm{m}$. Kleinebudde et al. also stress the importance of binder requirement stating that if binder levels are to be kept constant for varying degrees of MCC content the results are not directly 
Table 1

Physico-chemical characteristics for microcrystalline cellulose Avicel 105 and $\alpha$ lactose monohydrate.

\begin{tabular}{|c|c|c|c|c|c|}
\hline Powder & $\begin{array}{l}D_{50}[\mu \mathrm{m}] \\
\text { (volumetric) }\end{array}$ & $\begin{array}{l}\text { Span } \\
\left(D_{90}-D_{10}\right) / D_{50}\end{array}$ & Density $\left[\mathrm{kg} / \mathrm{m}^{3}\right]$ & $\begin{array}{l}\text { Solubility } \\
{\left[\mathrm{g} / 100 \mathrm{~cm}^{3} \text { water at } 25^{\circ} \mathrm{C}\right]}\end{array}$ & $\begin{array}{l}\text { Carr index } \\
\left(100\left(\rho_{T}-\rho_{B}\right) / \rho_{T}\right)\end{array}$ \\
\hline Microcrystalline cellulose & 20 & 2.0 & 1515 & 0 & 49 \\
\hline$\alpha$-Lactose monohydrate & 60 & 2.3 & 1510 & 17 & 52 \\
\hline
\end{tabular}

comparable. The studies of Leuenberger et al. [5] and Betz et al. [6] show that a ternary powder mixture composed of: $86 \%$ (of the dry mass) lactose, $10 \%$ corn starch and $4 \%$ Polyvinylpirrolidone granulated with water can allow reliable control of the granulation process. Mackaplow et al. [7] showed that for wet high shear granulation of three different grades of lactose (fine, medium, and coarse) with water that torque curves become less reproducible and present more noise with decreasing mean granule size of the starting materials. They found the granulation process for the finer particles (mean particle size of $39 \mu \mathrm{m}$ ) to be influenced by increased wall build-up affecting the torque curve in ways not related to intrinsic granule properties.

Even though many studies deal with the granulation of pharmaceutical product mixtures there are still few systematic studies that allow characterizing the influence of formulation parameters (proportion of different components in the mixtures) and the influence of mixer design. We have set out to study the effect of microcrystalline cellulose content in lactose-MCC mixtures on wet mass rheology, high shear wet granulation kinetics, wet mass consistency and dry granule strength. Additionally we will try to relate the observations to the starting materials' properties as well as attempt to validate our observations on two different scales. There is also an added complexity to this study as the powders chosen are fine (microcrystalline cellulose powder Avicel 105 with mean granule size of $20 \mu \mathrm{m}$ and a fine lactose grade, $\alpha$ lactose monohydrate with a mean granule size of $60 \mu \mathrm{m}$ ), very cohesive, presenting poor flowing characteristics. This makes them interesting candidates for size enlargement operations.

\section{Materials and methods}

\subsection{Materials}

Microcrystalline cellulose (Avicel PH 105, FMC Biopolymer) and $\alpha$ lactose monohydrate (Fischer Scientific) were used in this study. Some relevant properties for these materials are presented in Table 1. Solubility for the lactose grade is presented as indicated by the manufacturer, granule size distributions were determined using a laser particle size analyzer (Malvern Mastersizer, dry dispersion) while the densities needed for the calculation of the Carr index [8]

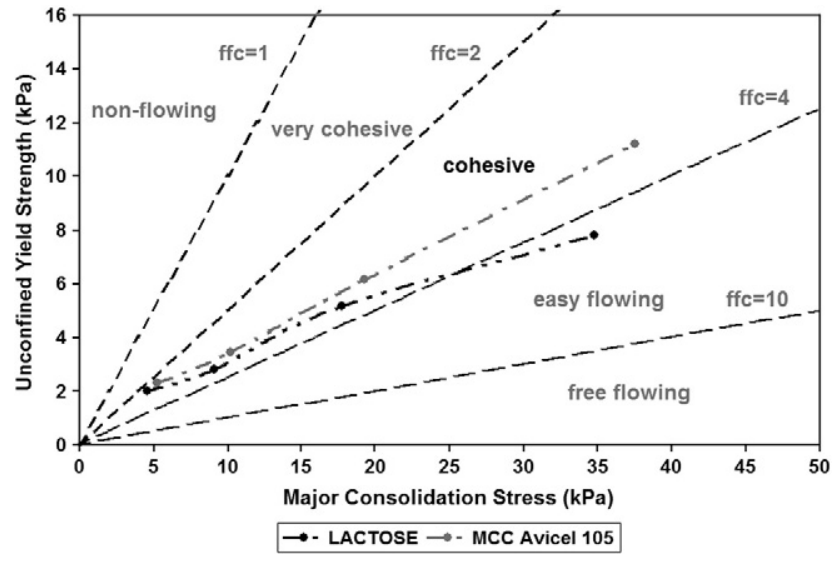

Fig. 1. Flow functions for the studied powders.

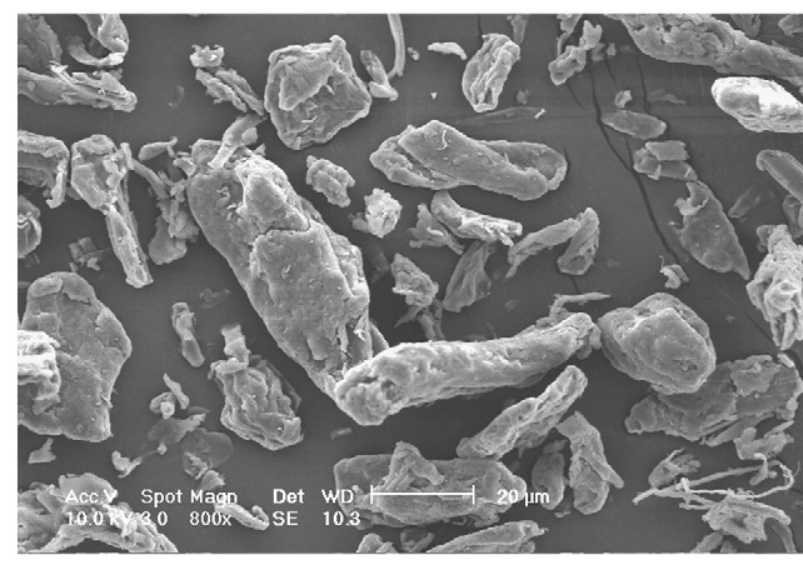

Fig. 2. SEM image of initial MCC Avicel 105 particles.

were obtained on a tapped density tester (Erweka Ltd). The liquid used for granulation was ultra pure water. As the mean granule size and Carr index show we are dealing with cohesive powders. Tests carried out on a Freeman FT4 equipped with a shear cell allowed us to obtain the flow functions for our initial powders following the Jenike
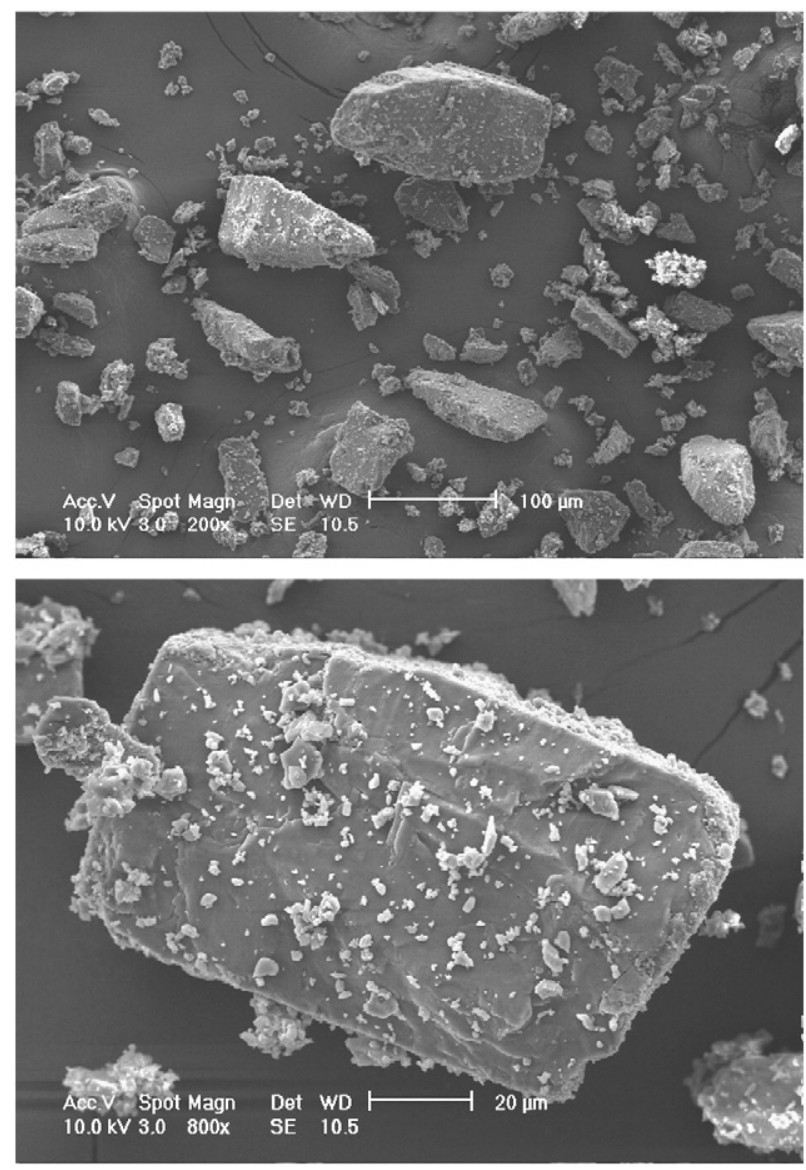

Fig. 3. SEM image of initial $\alpha$ lactose monohydrate particles. 
classification of flowability by flow index [9] and allowed us to further describe the cohesion of our initial powders (Fig. 1). Both powders are in the cohesive region while only for the highest major consolidation stress the flowability of the lactose powder is slightly improved.

Fig. 2 shows the scanning electron microscope images of initial MCC particles while Fig. 3 presents the initial lactose particles. The MCC (Fig. 2) presents itself as a fine, white, water insoluble powder showing particles with elongated, irregular form. The initial lactose particles (Fig. 3) show large lactose crystals with finer lactose particles sticking to their surface.

The mixture ratios are based on mass percentage calculations while the difference in bulk density between mixtures has been taken into account so as to ensure the same fill ratio in all experiments.

\subsection{Granulation equipment}

\subsubsection{Mi-Pro® high shear mixer}

The Mi-Pro (Pro-C-Ept, Zelzate, Belgium) high shear mixer allows granulating while recording real time impeller torque values with $1 \mathrm{~s}$ intervals. This vertical axis high shear granulator (Fig. 4a) was equipped with a $1.9 \mathrm{~L}$ glass bowl, granulation runs were carried out with the impeller rotating at $800 \mathrm{rpm}$ (impeller tip speed of $5.86 \mathrm{~m} / \mathrm{s}$ ) and with the chopper rotating at $3000 \mathrm{rpm}$ (impeller tip speed of $3.9 \mathrm{~m} / \mathrm{s}$ ). The powder bed was homogenized by dry mixing for $2 \mathrm{~min}$. This was considered sufficiently long to observe if any dry aggregation occurred and if any artifacts on the torque curve might appear. This was followed by water addition at a constant rate. Water addition was done in a drop-by-drop manner using a Dosimat 760 syringe pump

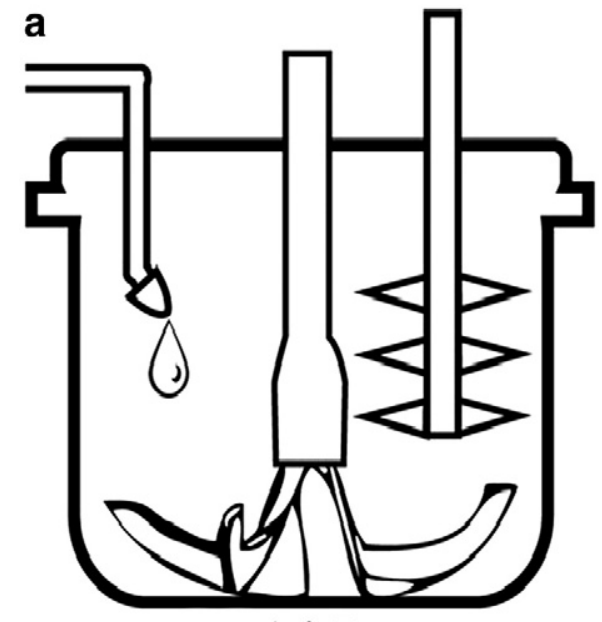

\section{$1.9 \mathrm{~L}$}

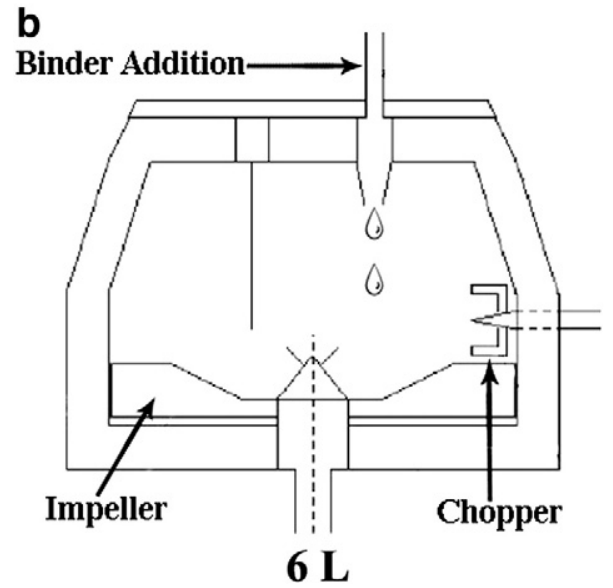

Fig. 4. Schematic representation of the Mi-Pro (a) and Diosna P6 (b) HSM.
(Metrohm, Berchem, Belgium). Operation was done through a computer whose software interface allows changing impeller, chopper and liquid dosing speed. It also allows the operator to follow a real time evolution of the recorded torque and temperature. Torque curves are represented using a normalized impeller torque which is the recorded torque from which a mean value of torque obtained with the same mixing speeds, impeller and chopper, on an empty bowl has been subtracted and the result is divided by the initial mass of dry powder. The fill ratio of the bowl is $26 \%$ (dry powder bulk volume to bowl volume) and the granulation time after the 2 min of dry mixing is $12 \mathrm{~min}$.

\subsubsection{Diosna $P 6 ®$ high shear mixer}

In order to validate our experiments on another scale the $6 \mathrm{~L}$ capacity Diosna ${ }^{\circledR}$ HSM (Fig. 4b) was used in this study. The same fill ratio and granulation times have been kept as on the Mi-Pro. The low position of the chopper in the Diosna high shear mixer, closer to the powder bed can lead to increased granule breakage. As a consequence the chopper on the Diosna has not been operated. In the Diosna HSM agitation is achieved by the means of a four-bladed impeller and a tulip-shaped chopper. Binder addition is carried out by a manually operated Masterflex Easy-Load II pump at the top of the bowl. Besides the differences in bowl geometry and chopper position there is also a difference in drop volume, the capillary in the case of the Mi-Pro is about $400 \mu \mathrm{m}$ while on the Masterflex the size is about $800 \mu \mathrm{m}$. Also while in the Mi-Pro binder addition is done in the direction of the chopper in order to help with the dispersion of the binder on the Diosna where such orientation is not possible.

a
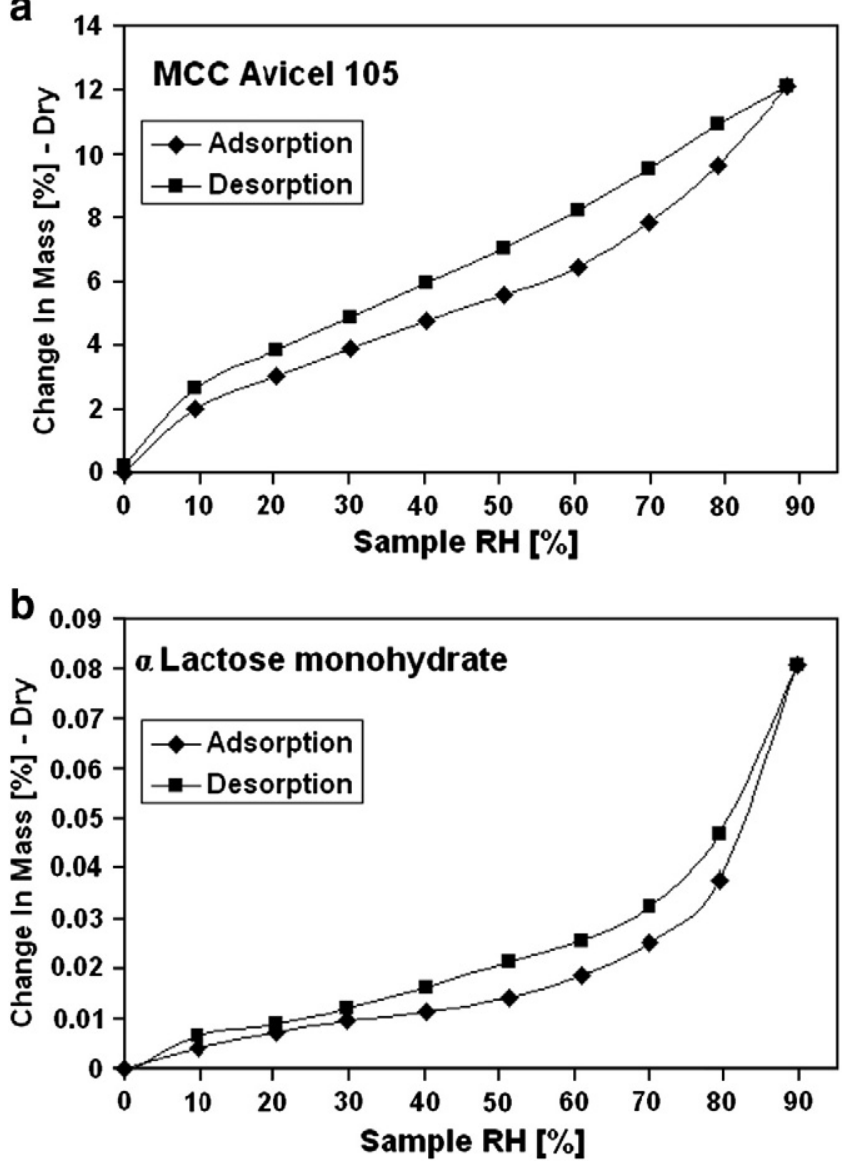

Fig. 5. Water sorption isotherms for the starting materials: MCC (a) and lactose (b). 
a
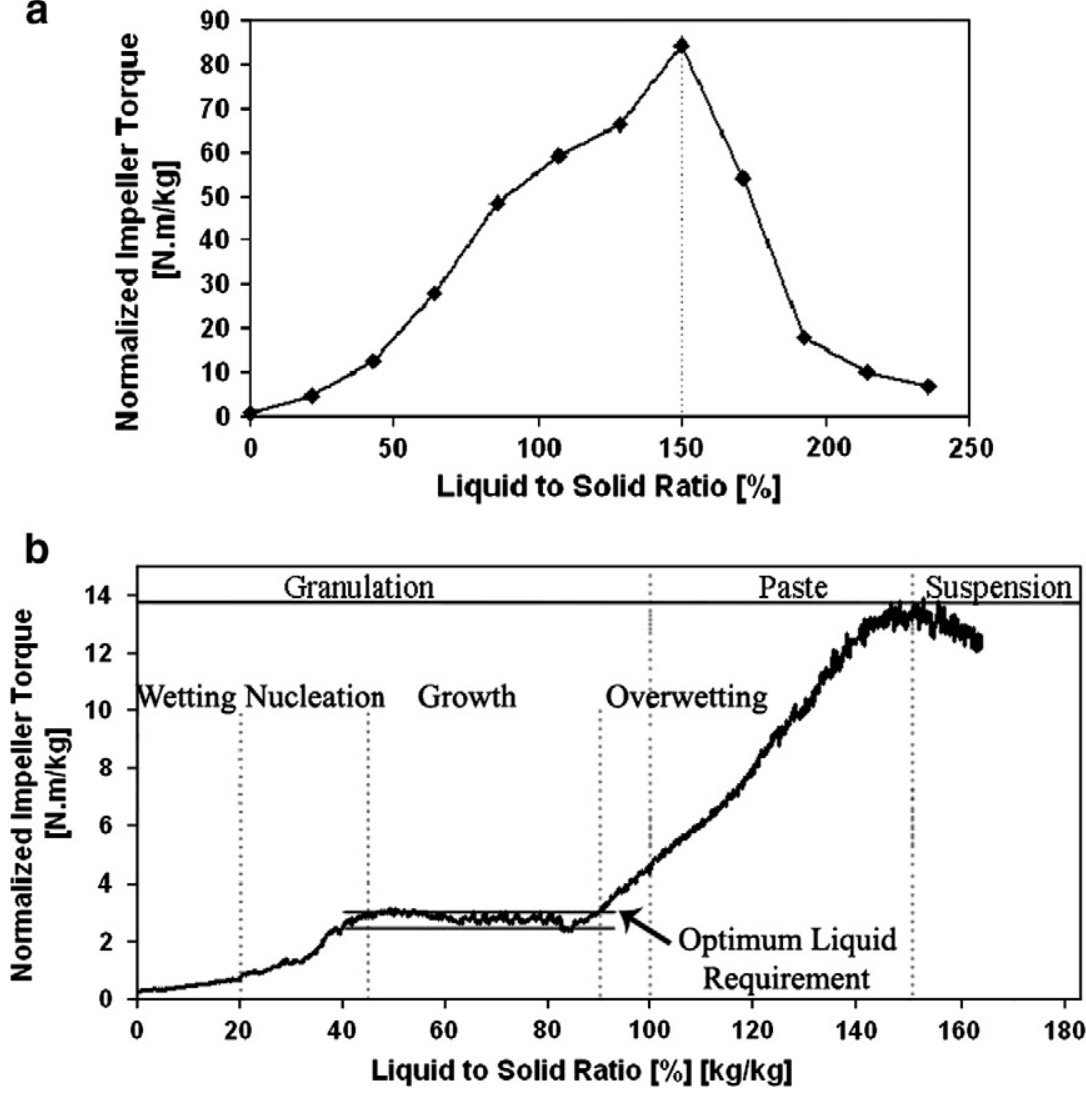

Fig. 6. Torque curves developed upon gradual addition of binder on the (a) MTR and (b) Mi-Pro for MCC.
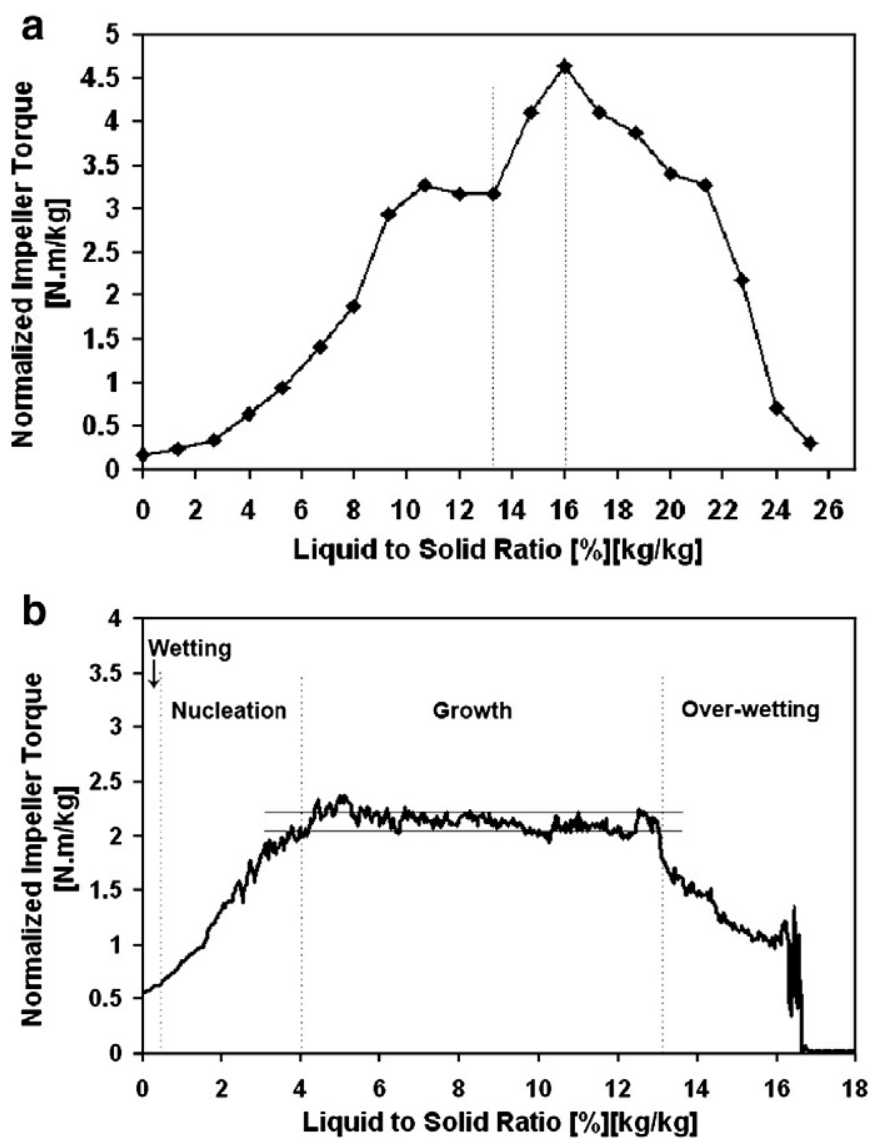

Fig. 7. Torque curves developed upon gradual addition of binder on the (a) MTR and (b) Mi-Pro for lactose.

\subsection{Characterization methods}

\subsubsection{Rheology}

In order to determine the wet mass rheology and wet mass consistency, a mixer torque rheometer (Model MTR, Caleva Ltd, Dorset, England) is used to measure the impeller torque developed. The MTR was used as a tool for initial characterization of the powder binder couple by carrying out the multiple binder addition test. The binder is added to the powder with a certain liquid flow rate and then both binder and powder are mixed for a certain time. This step is repeated a certain number of times usually taking the powder from the dry state to a suspension. The mixer torque rheometer measures two values in function of time and added liquid: the mean torque and the amplitude of the torque oscillations or torque range [10]. The mean torque is equivalent to the resistance of the wet mass to mixing and allows the monitoring of the different states of saturation of the agglomerate. The torque range describes the heterogeneity of the wet powder being a measure of mean torque standard deviation. In this study only the mean torque is represented as the mean range showed the same effects.

The dry powder was mixed in the rheometer for $30 \mathrm{~s}$ in order to obtain the baseline response. The quantity of dry powder was selected so as to cover the mixing blades which led to a fill ratio of about $40 \%$. The binder was added by the multiple addition method. After each addition the wet mass is mixed and at the end of the mixing period a mean torque value is stored so that each point on the torque curve corresponds to an addition and so to a new saturation value of the wet mass. The torque values are expressed as normalized torque thus taking into account the initial fill level. For all our experiments on the mixer torque rheometer a target mixing time of $10 \mathrm{~min}$ has been sought in order to ensure the good distribution of the binder in the powder bed. 
The wet mass consistency was also determined on the MTR. Samples of wet granules were mixed in the MTR and the torque response is recorded. The volume of the wet mass sample was chosen so as to cover the impeller blades (fill ratio of $40 \%$ ). A time of $480 \mathrm{~s}$ was imposed for mixing of the wet granules. Granules are deformed under the action of the impellers and torque values evolve to a constant value named wet mass consistency.

\subsubsection{Granulation kinetics}

In order to assess granule growth and morphological properties representative samples of solid ( 15 to $30 \mathrm{~g}$ ) were removed from the bed and tray-dried at $40{ }^{\circ} \mathrm{C}$ over night for approximately $24 \mathrm{~h}$. Due to the small size of the Mi-Pro mixer bowl each sample was taken from a new batch with experiments being carried out twice. The dried granules were sieved and 15 size fractions were collected. Weight mean diameter was determined using the following equation:

Mean diameter $: D_{\mathrm{pm}}=\frac{\sum_{i} f_{i} \cdot d_{p i}}{\sum_{i} f_{i}}$

where $f_{i}$ is the particle mass fraction of size interval $i, d_{p i}$ is the mean diameter of size interval $i(\mu \mathrm{m})$. For a better understanding of agglomeration mechanisms, three characteristic granule classes were
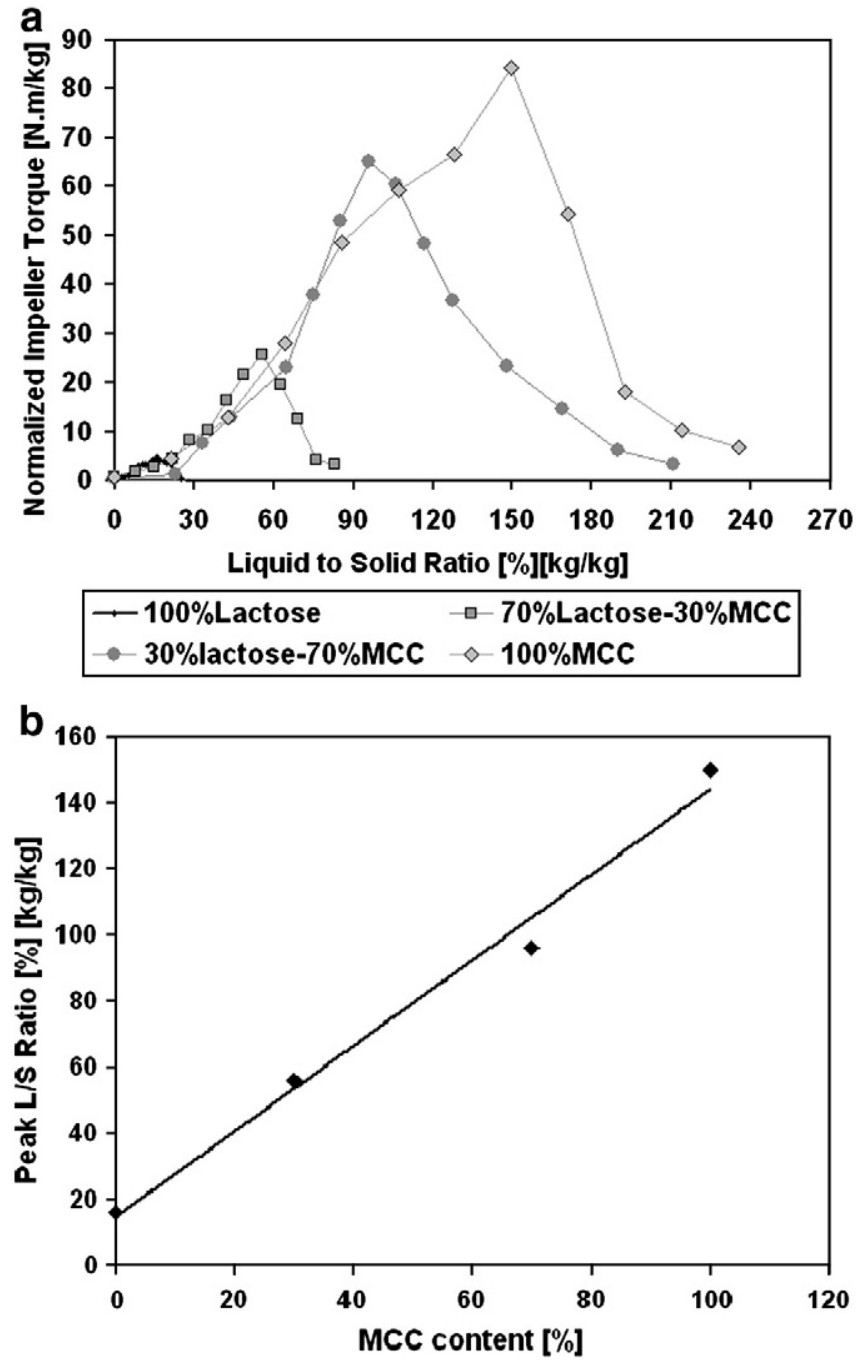

Fig. 8. MTR torque curves developed upon gradual addition of binder for the pure starting materials and the studied formulations (a) and peak $\mathrm{L} / \mathrm{S}$ ratio as a function of MCC content (b) defined: fine (corresponding to the initial powder with diameters inferior to $200 \mu \mathrm{m}$ ), intermediate (weak granules with diameters between $200 \mu \mathrm{m}$ and $800 \mu \mathrm{m}$ ) and coarse agglomerate (strong, dense granules with diameters greater than $800 \mu \mathrm{m}$ but smaller than $5 \mathrm{~mm}$ ). Granules exceeding $5 \mathrm{~mm}$ are defined as lumps.

Kinetic results are expressed in function of the fraction added liquid to initial dry powder mass named liquid to solid ratio:

$L /$ SRatio $=\frac{\text { Added liquidmass }[\mathrm{kg}]}{\text { Initialpowder mass }[\mathrm{kg}]} * 100(\%)$

Discussing kinetics as a function of added liquid or as liquid saturation (as defined in different studies $[2,5]$ ) is debatable. With products like microcrystalline cellulose where liquid solid interaction involves swelling (Luukkonen et al. [11]) and formation of a crystallite gel during pelletization (Kleinebudde et al. [12]) calculating saturation is extremely difficult. Also the presence of water soluble lactose in the mixture can increase the liquid to solid ratio if lactose goes into solution. However as discussed by Ramaker [13] the presence of MCC that can absorb large amounts of binder can limit lactose solubility.

\subsubsection{Dry granule strength}

An Instron ${ }^{\circledR} 5567$ press has been used in order to assess dry granule strength by single granule uniaxial compression tests, equipped with a $500 \mathrm{~N}$ load cell. Single granules $(n=30$ granules for each formulation) were compressed with a stainless steel probe with a diameter of $10 \mathrm{~mm}$. Granules tested were the granules with the diameter close to the mean diameter of the granulation run. Granule
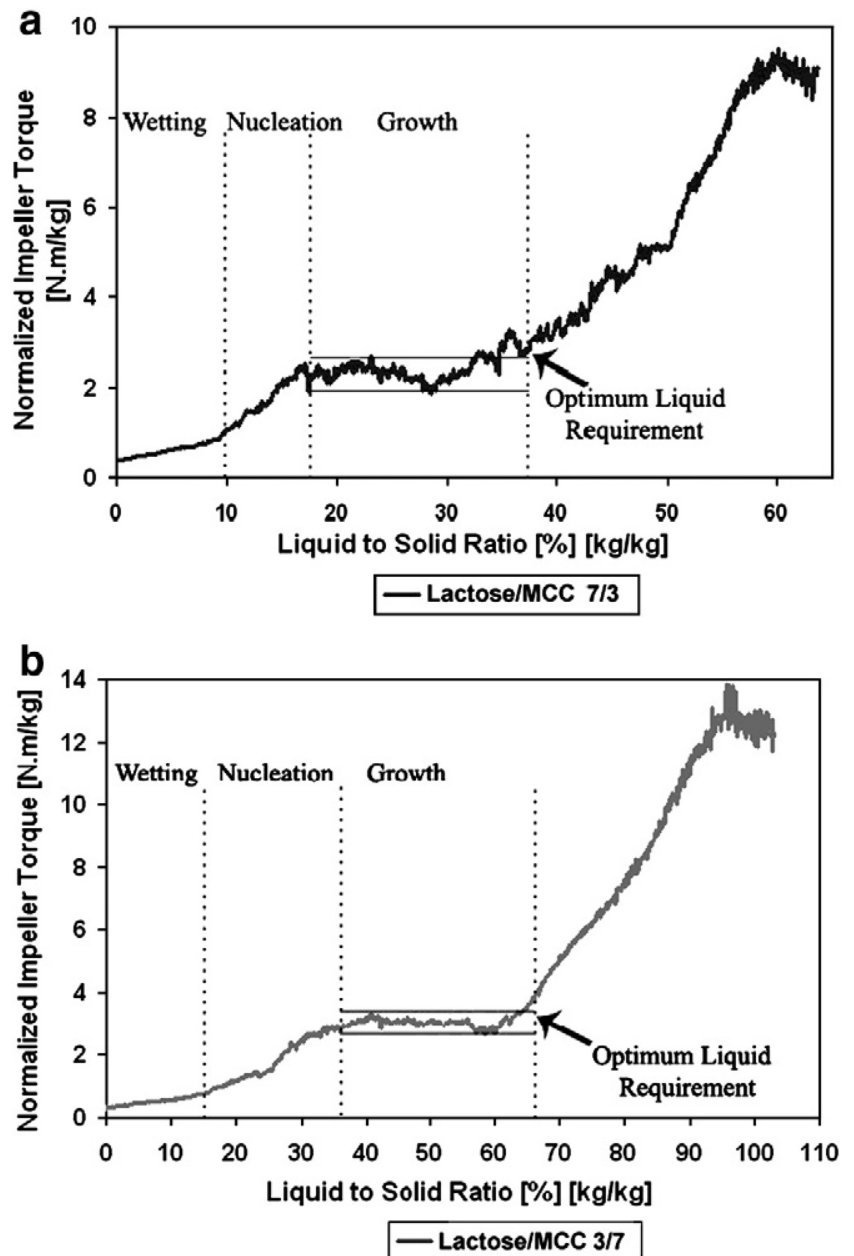

Fig. 9. Torque curves on the Mi-Pro HSM for the Lactose/MCC 7/3 (a) and the Lactose/ MCC 3/7 (b) formulations. 

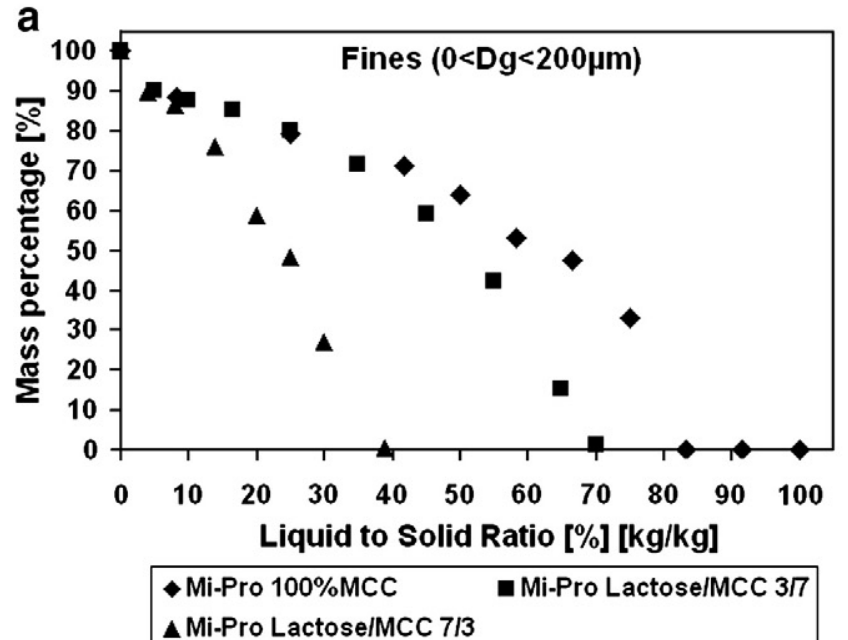

C

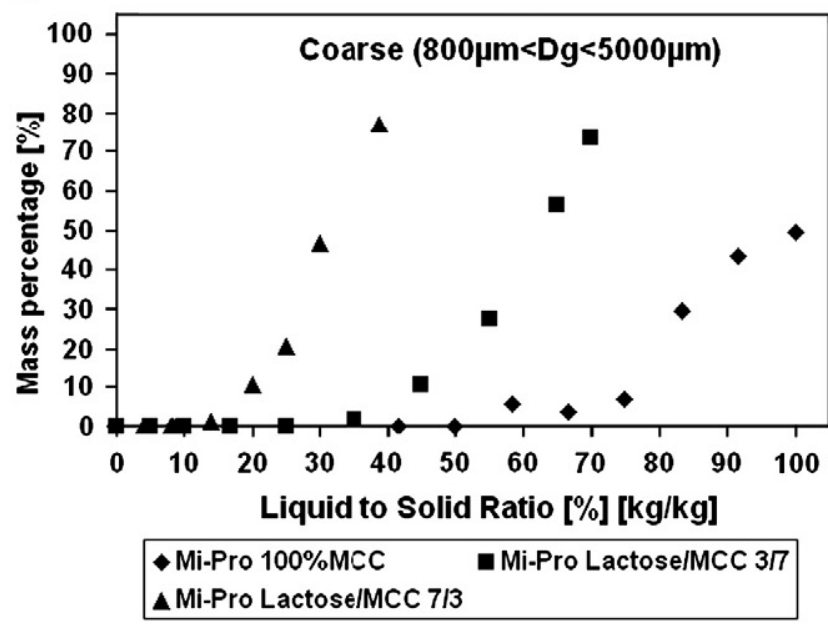

b

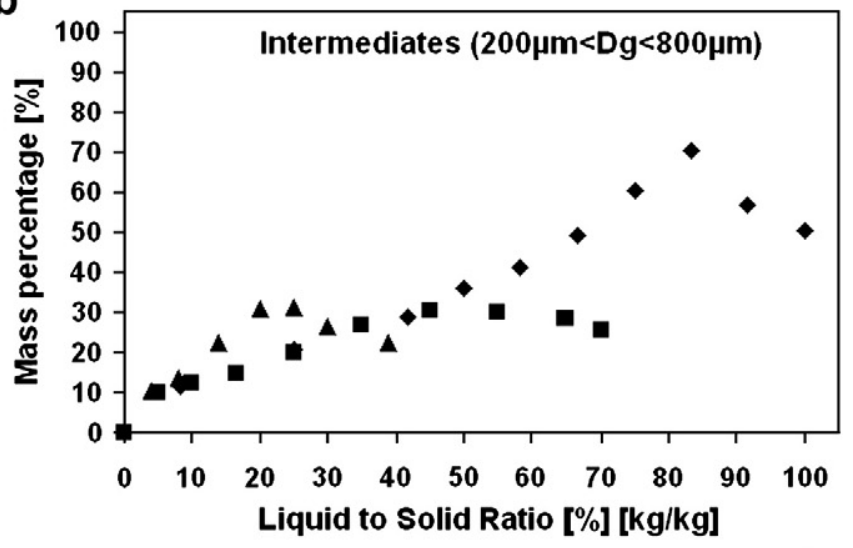

- Mi-Pro $100 \%$ MCC $\Delta$ Mi-Pro Lactose/MCC $7 / 3$

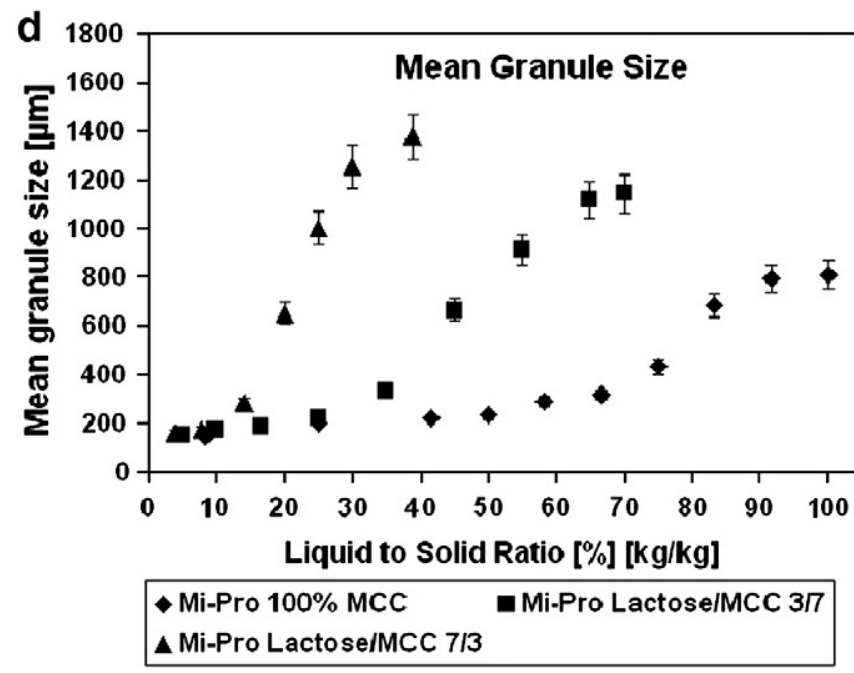

Fig. 10. Evolution of fine (a), intermediate (b) and coarse(c) size classes and mean granule size for the studied formulations (d) using $100 \%$ MCC as reference.

strength was assessed using the following equation proposed by Adams et al. [14]:

$\sigma=4 \frac{F_{\max }}{\pi \cdot D^{2}}$

where $F_{\max }$ is the fracture force and $D$ is the mean granule diameter. Imposed velocity of the steel probe was $0.1 \mathrm{~mm} / \mathrm{s}$. Because granules are semi-brittle measuring the compression strength is possible. When the granule is subjected to compressive strain (displacement) a marked drop in the signal can be observed when the granule first cracks (at $F_{\max }$ ). The values recorded after this point on the loaddisplacement curve are of little practical use as they represent the compression of granule fragments and eventually powder particles.

\subsubsection{Dynamic water sorption system}

Adsorption isotherms, characterizing liquid to solid adsorption have been investigated for the starting materials. They represent the relationship between the amount of gas or liquid adsorbed by unit mass of solid and the equilibrium pressure (or relative pressure) at a known temperature.

The sorption characteristics of cellulose and lactose have been determined by a gravimetric method using a Surface Measurement Systems ${ }^{\circledR}$ automated Dynamic Vapor Sorption (DVS-1000) instrument. It measures the uptake and loss of vapor using a controlled atmosphere microbalance with a mass resolution of $\pm 0.1 \mu \mathrm{g}$ equipped with a video microscope system. The vapor partial pressure around the sample is controlled by mixing dry and saturated vapor gas flows using electronic mass flow controllers. In these experiments, the variation of mass of the sample was measured as a function of time over a range of values of relative humidity. The temperature is maintained constant at $25^{\circ} \mathrm{C} \pm 0.1^{\circ} \mathrm{C}$ by enclosing the system in a temperature-controlled incubator.

\subsubsection{Morphological characterization}

The dried agglomerates obtained during the granulation are analyzed by a FEI scanning electron microscope. The granules were coated with gold using coating apparatus to create electric conductivity on the surface of the samples.

\section{Results and discussion}

\subsection{Characterization of the starting materials}

Differences in liquid requirement between MCC and lactose have been apparent from the first preliminary characterization studies on the mixer torque rheometer and the water sorption isotherms. Water sorption isotherms (Fig. 5a and b) show type II sorption isotherms (Rouquerol et al. [15]). The adsorption of the first layer of water molecules on the particle surface can be identified by the first inflexion point in the sorption curve. With increasing relative humidity multiple layers of water are being adsorbed to the surface 
and as a second inflexion point appears in the curve water becomes loosely bound and mobile, with minimal water-solid interactions. MCC presents a much higher water affinity than lactose. For the same values of sample relative humidity, for instance for $80 \%$ when water is loosely bound MCC adsorbs about $8 \%$ while lactose adsorbs only about $0.04 \%$. This result also implies that when granulating liquid would be present in a loose form at the particle surface much quicker for the lactose particles than for the MCC particles.

Fig. 6 shows the torque curve obtained on the MTR (Fig. 6a) and the torque curve obtained on the Mi-Pro (Fig. 6b) for the MCC powder. Fig. 7 gives the same information for lactose. The peak of the torque curve for the MCC powder corresponds to the wet powder mass turning to a highly cohesive paste in the Mi-Pro. The end of the plateau region in Fig. $6 \mathrm{~b}$ has been found to correspond to the total consumption of the fine particle class [16]. Granulation can still be continued in order to maximize the coarse granules class up to a $\mathrm{L} / \mathrm{S}$ ratio of $100 \%$. For values above $100 \% \mathrm{~L} / \mathrm{S}$ ratio overwetting occurs. Growth mechanisms proposed in Figs. 6b and 7b are based on characteristic granule class evolution and SEM observations. For the lactose MTR curve a shoulder is observed before the torque peak appearing at $16 \% \mathrm{~L} / \mathrm{S}$ ratio. Benali [17] showed that for the same type of powder (from the same source) the optimum $\mathrm{L} /$ $S$ ratio of $13 \%$ would correspond to the end of the plateau region observed on the lactose MTR curve. Our experiments on the Mi-Pro with lactose have shown a very strong interaction between the wet mass and the mixer bowl leading to large wall build-ups for $\mathrm{L} / \mathrm{S}$ ratios exceeding $4 \%$. Stopping the granulation experiment and scraping the mixer bowls allow granulation to a similar L/S ratio as that observed by Benali [17]. With the gradual addition of binder, the end of the plateau region on the Mi-Pro (Fig. 7b) corresponds to the majority of the wet mass exiting the impeller action and sticking to the mixer wall and a small fraction of product evolving to a suspension. It is our opinion that for the granulation of pure lactose a better granulation process can be achieved with the whole optimum water requirement added over a short period of time in the beginning of the granulation. This would allow the formation of an initial homogenous paste like mass that would be further dispersed by the granulating equipment and shaped into granules.

Comparing the rheological data for the two starting materials we can conclude that:

- lactose requires less binder to granulate than microcrystalline cellulose (13\% vs $100 \%$ );

- agglomerated MCC particles develop a far greater cohesion than agglomerated lactose particles on the MTR (Figs. 6a and 7a);

- torque curves on the Mi-Pro for MCC and lactose are not completely comparable since the process is less than optimal for lactose granulation after the plateau region. However, what is
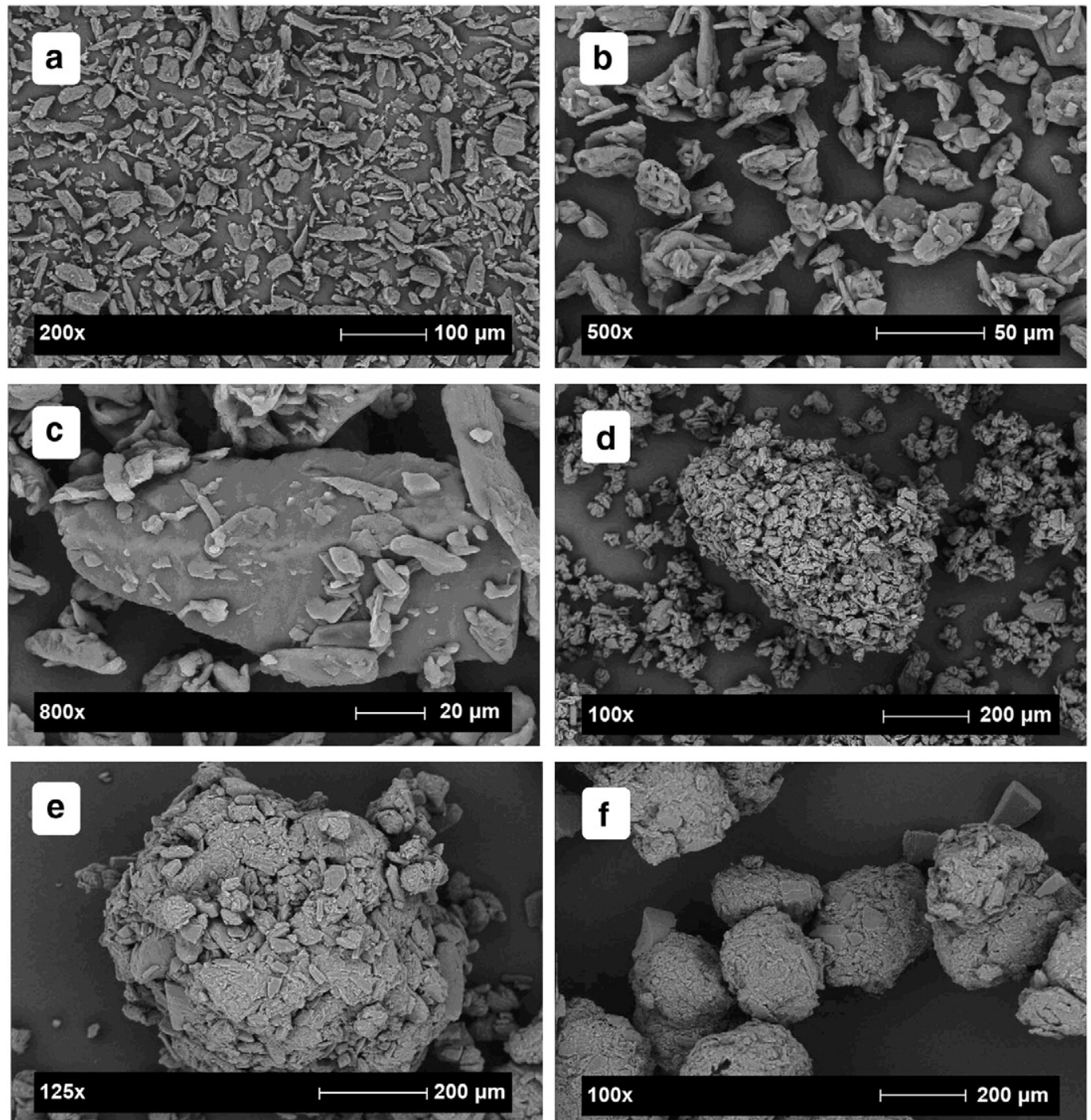

Fig. 11. SEM images of agglomerates at different L/S ratios for the Lactose/MCC 3/7formulation: (a) wetting (L/S=15\%), (b) nucleation (L/S=25\%), (c) MCC particles sticking on lactose particles ( $\mathrm{L} / \mathrm{S}=25 \%)$, (d) growth $(\mathrm{L} / \mathrm{S}=40 \%)$, (e) growth $(\mathrm{L} / \mathrm{S}=55 \%)$, (f) final granules $(\mathrm{L} / \mathrm{S}=68 \%)$. 

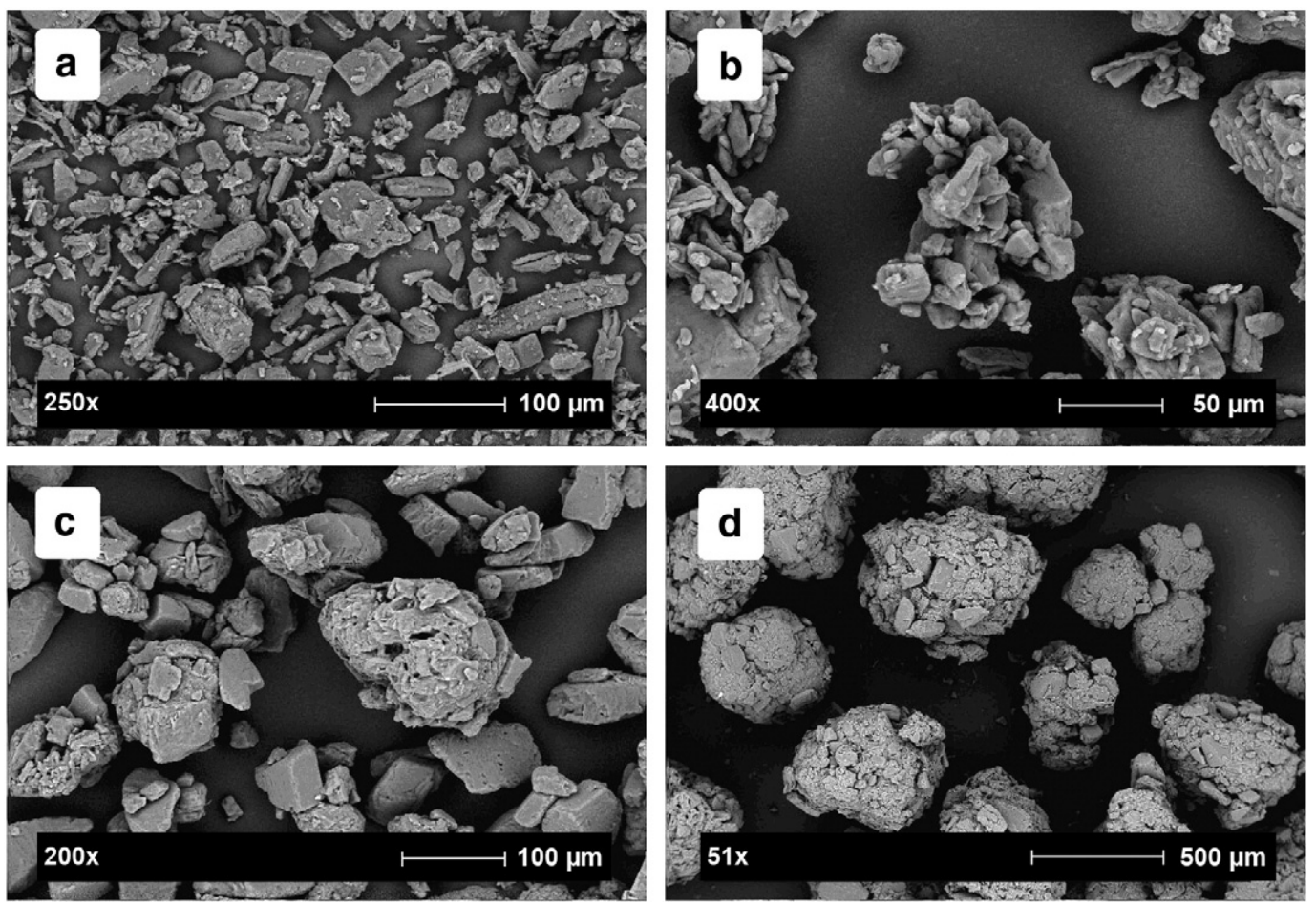

Fig. 12. SEM images of agglomerates at different $L / S$ ratios for the Lactose/MCC $7 / 3$ formulation: (a) wetting (L/S $=10 \%)$, (b) nucleation (L/S = 16\%), (c) growth (L/S = 32\%), (d) final granules $(\mathrm{L} / \mathrm{S}=38 \%)$.

evident, is the much shorter wetting region for the lactose particles. This confirms our predictions from the water sorption isotherms, that lactose will present binder on the surface for lower binder contents, promoting nucleation and growth.

\subsection{Powder mixture characterization}

In order to study the granulation of powder mixtures we chose two different formulations, one containing 7 parts lactose and 3 parts MCC Avicel 105 called "Lactose/MCC 7/3" and one containing 7 parts MCC and 3 parts lactose called "Lactose/MCC 3/7". We compared them in terms of rheological characterization, granule growth kinetics, wet mass consistency and dry granule strength.

\subsubsection{Rheological characterization of the mixtures}

Besides the difference in liquid requirement for the starting materials there is also a difference in terms of developed torque: 16 times higher for MCC than lactose. Fig. 8a shows the MTR curve for the two studied formulations as well as the curves for the pure starting materials. We find that increasing the percentage of microcrystalline cellulose increases linearly the liquid requirement for the mixture at the peak torque (Fig. 8b). As expected from the findings of Kristensen et al. [1] the liquid requirement and torque increase with increasing MCC percentage.

\subsubsection{Granulation kinetics for the studied formulations}

In order to obtain reproducible results granulation is best stopped in the passage from the pendular to the funicular saturation stage which is defined by the plateau region on the torque curves (Leuenberger [5]). Stopping liquid addition at L/S ratios closer to the end of the plateau allows obtaining denser granules, while slightly increased values allow a reduction of fines in the system but in the same time endanger overwetting. For the studied formulations values closest to the end of the plateau have been chosen as optimum liquid

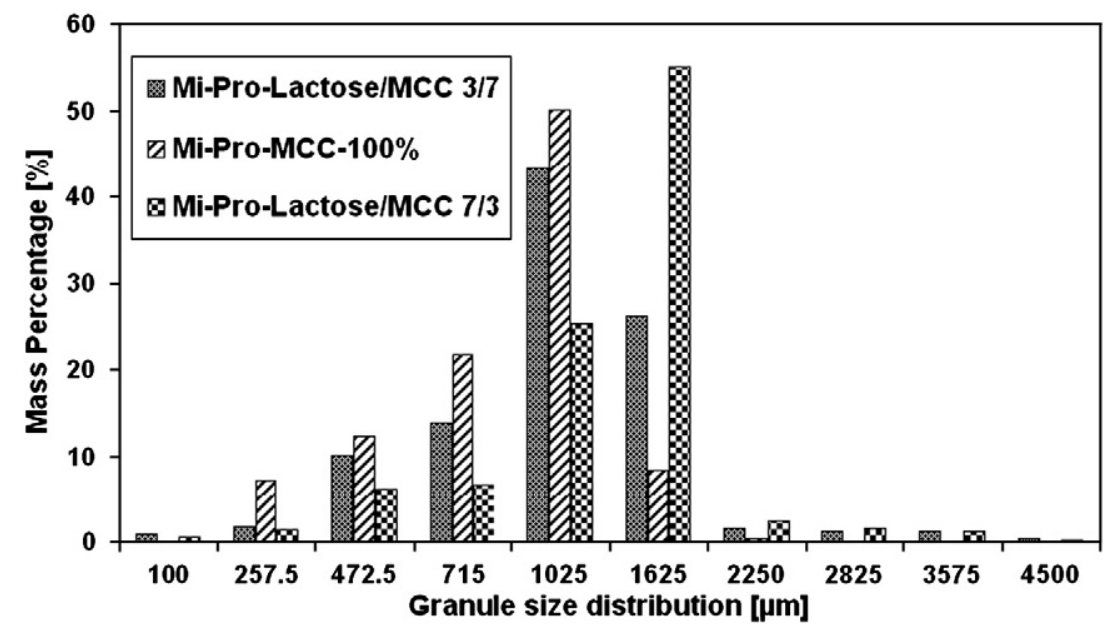

Fig. 13. Granule size distribution for the two studied formulations as well as $100 \%$ MCC. 
requirements for granulation. This corresponds to 38\% for the granulation of the Lactose/MCC $7 / 3$ formulation (Fig. 9a) and $68 \%$ for the granulation of the Lactose/MCC 3/7 formulation (Fig. 9b). From an optimum liquid requirement point of view this values fall as expected in between the liquid requirement for pure lactose granulation at $13 \% \mathrm{~L} / \mathrm{S}$ ratio and pure MCC at $100 \% \mathrm{~L} / \mathrm{S}$ ratio. It can also be observed that torque curves have a similar profile to the one presented for pure MCC in Fig. $6 \mathrm{~b}$.

Torque profiles are similar for both formulations: after an initial wetting period longer for the Lactose/MCC $3 / 7$ formulation the characteristic S-shaped "turning point" (as defined by Betz et al. [6]) can be observed. It signals the pendular saturation stage where nucleation of initial particles usually occurs. This is followed by the plateau region when growth of the particles occurs in the funicular saturation stage (Goldszal and Bousquet [18]). Torque values on the plateau are slightly higher for the Lactose/MCC 3/7 formulation reflecting the higher resistance to mixing of the MCC and indicating greater resistance to impact and shear. It can also be observed that the Lactose/MCC 7/3 formulation torque curve is noisier as agglomerates containing more lactose are stickier and can easily adhere to the glass bowl causing inhomogeneous flow of the wetted material. This phenomenon has also been observed by Holm et al. [4].

The evolution of the characteristic size classes allows us to confirm the interpretation of the obtained torque curves. The granule growth kinetics for the two formulations are compared using 100\% MCC as reference (Fig. 10). Increasing lactose content in the mixtures decreases optimum water requirements and accelerates granule growth. Changes in growth mechanisms occur for similar values of $\mathrm{L} / \mathrm{S}$ ratio as observed in the torque curve (also confirmed by SEM observations).

An initial wetting period ranging from 0 to $10 \% \mathrm{~L} / \mathrm{S}$ ratio for the Lactose/MCC $7 / 3$ formulation and from 0 to $15 \%$ for the Lactose/MCC $3 / 7$ formulation is observed. At this stage no significant changes in the granule size distributions are observed. For higher $\mathrm{L} / \mathrm{S}$ ratios nucleation occurs marked by a sudden decrease in the fine particle class mass percentage (Fig. 10a).

The end of the nucleation stage can be observed as a change in the slope of the intermediate granules mass percentage (Fig. 10b). For $100 \%$ MCC growth can be observed in two stages:

- as a sudden increase in slope from 58\% L/S ratio to $83 \%$ corresponding to the preferred agglomeration of fine and intermediate granules until the fines are depleted,

- and agglomeration between intermediates, as a second stage, between $83 \%$ and $100 \%$. The intermediate particle class is never depleted as the high impeller and chopper speeds impose final granule sizes close to the upper boundary of the intermediates fraction $(800 \mu \mathrm{m})$.

For the Lactose/MCC $7 / 3$ and Lactose/MCC $3 / 7$ formulations nucleation occurs up to $20 \% \mathrm{~L} / \mathrm{S}$ ratio and up to $40 \%$ respectively. Nucleation end can be observed as a peak in intermediate particle class (Fig. 10b). This point corresponds to the beginning of the plateau region on the torque curves (Fig. 9). Alternatively this point also corresponds to the apparition of the first coarse granules in the system (Fig. 10c). This can explain why the intermediate granules' mass percentage slightly decreases. For the mixtures growth occurs by

Table 2

Wet mass consistencies and dry granule strengths for the two studied formulations and pure MCC ((Mi-Pro, $\mathrm{w}=800 \mathrm{rpm}, \mathrm{ch}=3000 \mathrm{rpm})$.

\begin{tabular}{lcc}
\hline Product & $\begin{array}{l}\text { Wet mass consistency } \\
{[\mathrm{N} \mathrm{m} / \mathrm{kg}]}\end{array}$ & $\begin{array}{l}\text { Granule strength } \\
{[\mathrm{MPa}]}\end{array}$ \\
\hline Lactose/MCC $7 / 3$ & $3.11 \pm 0.71$ & $18.1 \pm 3.29$ \\
Lactose/MCC 3/7 & $4.15 \pm 0.72$ & $26.46 .1 \pm 5.5$ \\
100\% MCC & $8.2 \pm 1.53$ & $32.1 \pm 5.8$ \\
\hline
\end{tabular}

agglomeration between fines and intermediates and also between intermediate granules.

For $\mathrm{L} / \mathrm{S}$ ratios increasing beyond $38 \%$ for the Lactose/MCC $7 / 3$ formulation and beyond $68 \%$ for the Lactose/MCC $3 / 7$ formulation granules become overwetted and uncontrollable growth takes place. The optimum liquid requirement is being well indicated by the end of the plateau on the torque curves. These results confirm the predictions made from torque curve analysis in terms of optimum liquid to solid ratio as well as separation between stages.

Fig. 10d presents the evolution of the mean granule size as a function of L/S ratio. Given the extent of the coarse particle class ( $800 \mu \mathrm{m}$ to $4 \mathrm{~mm}$ ) the mean granule size follows a similar evolution to that of the coarse particle size class. It is also interesting to note that in order to obtain granules of a certain size less binder is necessary with decreasing MCC content. Mean granule size, which increases with increasing lactose percentage in the mixtures, can be explained by the higher plasticity granted by the increased lactose content favoring coalescence between granules. Also, upon drying, granules containing MCC have been known to shrink (Kleinebudde et al. [19]).

\subsubsection{Morphology evolution during the wet granulation process}

SEM image analysis (Figs. 11 and 12) allows us to better understand the before mentioned granulation mechanisms. For low $\mathrm{L} / \mathrm{S}$ ratios we can see that no agglomerates are formed (Figs. 11a and 12a). For increased L/S ratios nucleation (small agglomerates of several initial particles) can be observed, this seems to occur preferentially between MCC particles (Figs. 11b and 12b) however the absence of fine lactose particles that most probably have gone into the solution should also be noted.

Given the behavior observed on the water sorption isotherms with lactose presenting loosely bound water for low masses of adsorbed water it is also reasonable to suspect that nucleation is accelerated by the presence of lactose, with MCC nuclei forming around lactose crystals. Fig. 11c shows to that effect MCC particles sticking to the surface of the larger lactose particles. For the Lactose/MCC 3/7 formulation growth occurs in two stages that don't seem to have an impact on the characteristic sizes evolution. First we can observe the agglomeration of MCC nuclei into MCC granules (Fig. 11d) for L/S ratios between 40 and $55 \%$ although they may still present a lactose core. This is followed by agglomeration between both MCC and lactose particles (Fig. 11e). The same agglomeration between MCC granules and lactose particles can also be observed for the Lactose/MCC $7 / 3$ formulation (Fig. 11c). Final granules (Figs. 11f and 12d) are spherical while initial particles, lactose crystals in particular, can still be observed.

The torque curves obtained on the Mi-Pro similar in nature to the pure MCC torque curve and SEM observations seem to imply that for

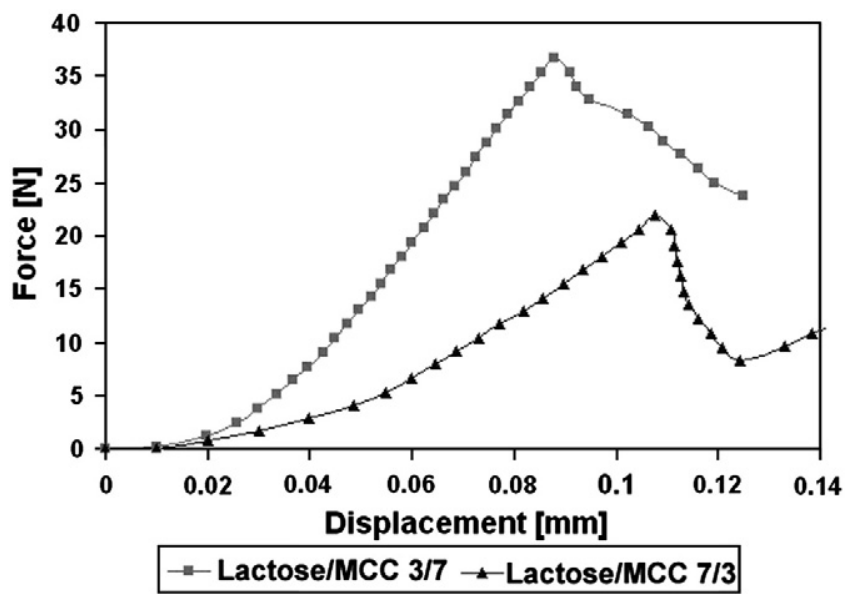

Fig. 14. Force-displacement curves for the lactose and MCC formulations. 
both formulations MCC controls the liquid distribution in the wet mass. These findings seem to relate favorably with the findings of Kuentz and Leuenberger [20] who showed that for a binary mixture of MCC and paracetamol the percolation threshold for MCC was about $20 \%$. This means that for values above $20 \%$ in the mixture MCC forms a continuous network. The results also confirm the assumption made in the beginning of this study that MCC would control binder distribution based on the water sorption isotherms given the higher affinity of MCC for water.

Fig. 13 shows the granule size distribution for pure MCC and the two studied formulations. No change in granule size distributions that form with varying formulation is observed. Increasing lactose content reduces the amount of finer particles and shifts the distributions to larger sizes. The larger amount of fines with increasing MCC content
Table 3

Mean granule size for the studied formulations on the two high shear mixers: Mi-Pro and Diosna.

\begin{tabular}{lc}
\hline Setup & $\begin{array}{l}D_{\text {pm }}[\mu \mathrm{m}] \\
\text { (volumetric) }\end{array}$ \\
\hline Mi-Pro & 1142 \\
Lactose/MCC 3/7 & 1376 \\
Lactose/MCC 7/3 & \\
Diosna - impeller tip speed & 1162 \\
Lactose/MCC 3/7 & 1249 \\
Lactose/MCC 7/3 & \\
Diosna - Froude & 1050 \\
Lactose/MCC 3/7 & 1132 \\
Lactose/MCC 7/3 & \\
\hline
\end{tabular}
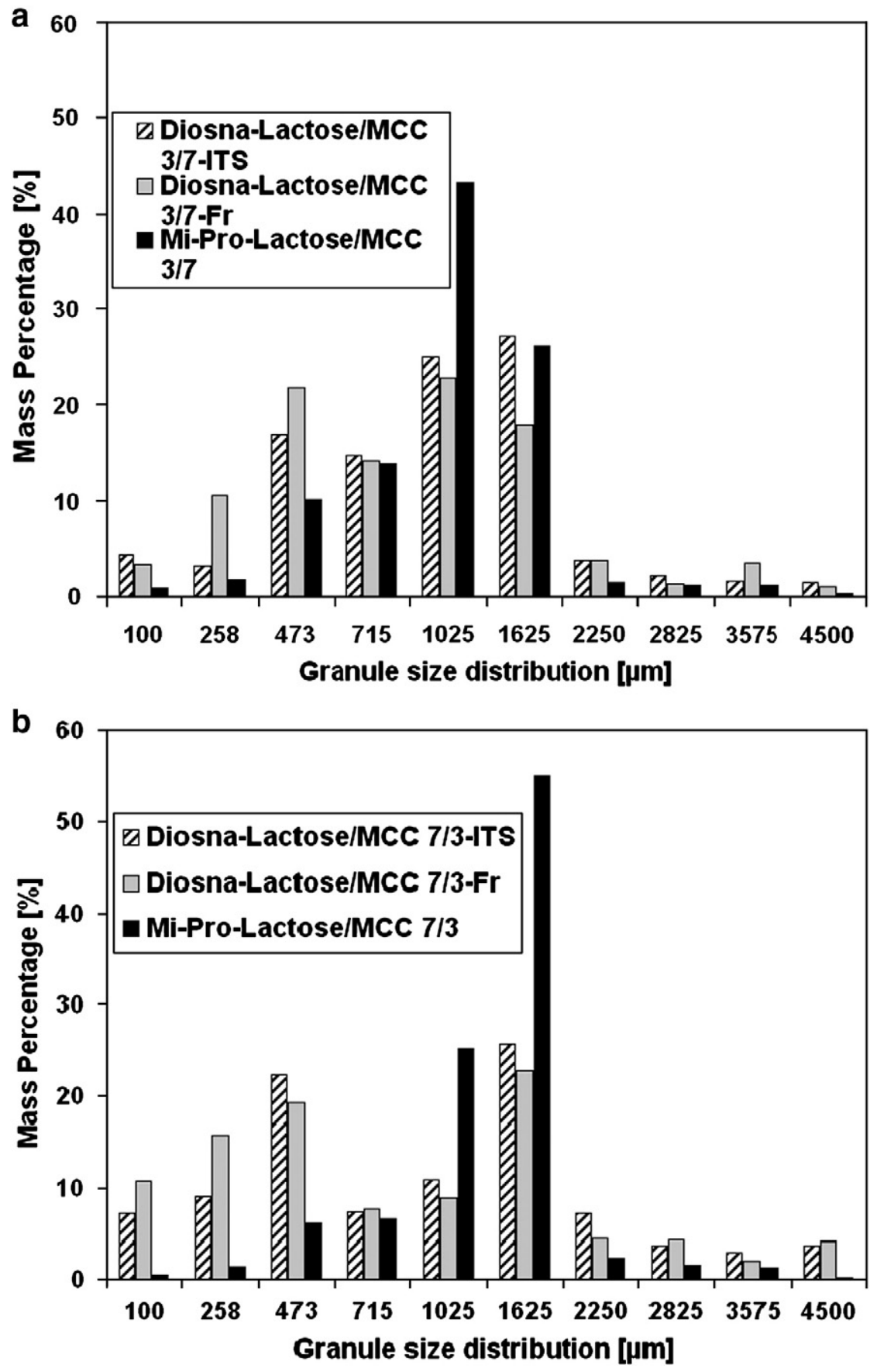

Fig. 15. Granule size distributions for the (a) Lactose/MCC $3 / 7$ formulation and (b) Lactose/MCC 7/3 formulation. 


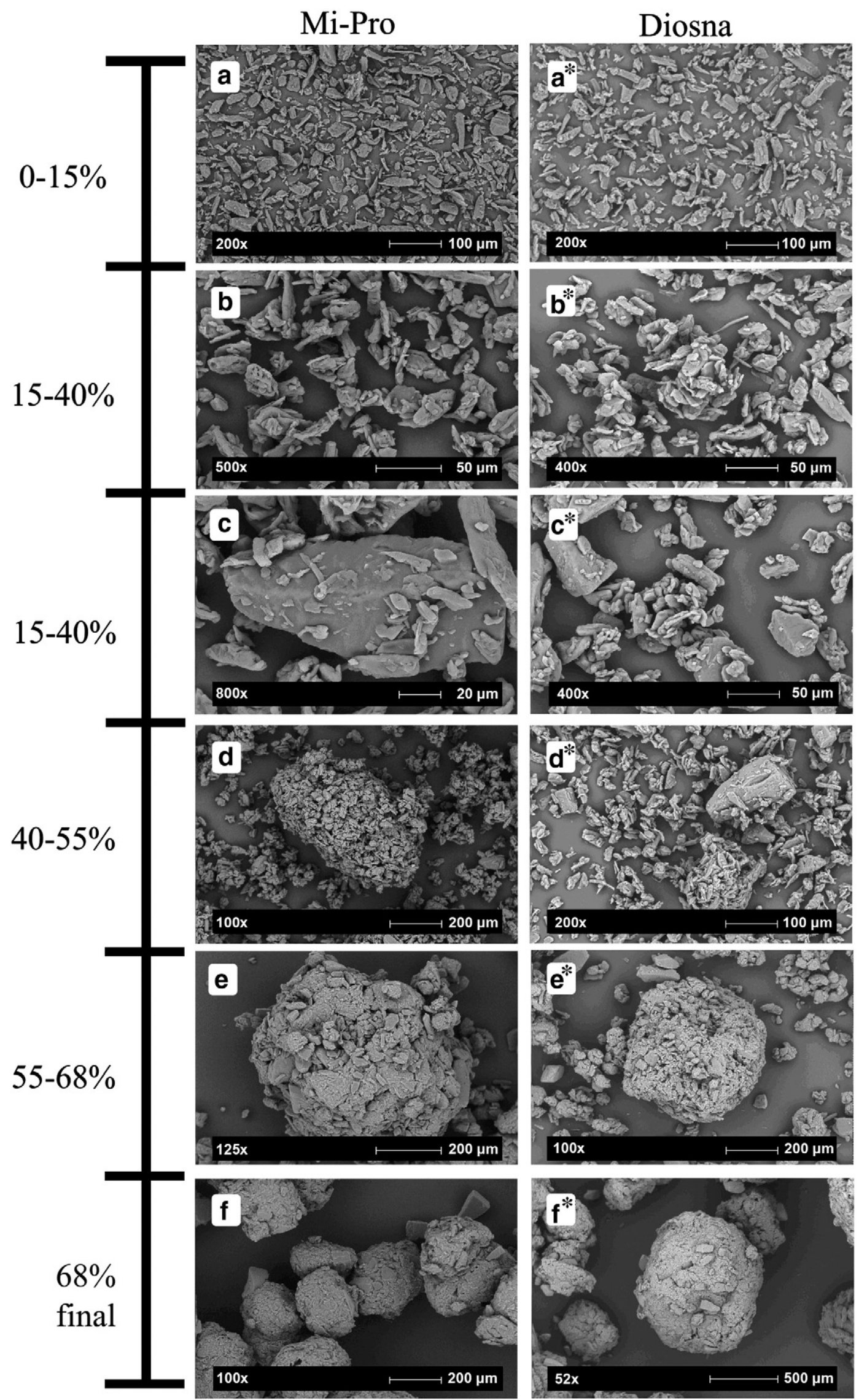

Fig. 16. Growth mechanisms on the Mi-Pro (a-f) and Diosna $\left(a^{*}-f^{*}\right)$ HSM for the Lactose/MCC $7 / 3$ formulation. 
Table 4

Wet mass consistencies and dry granule strengths for the different formulations on the Mi-Pro and the Diosna high shear mixers.

\begin{tabular}{llc}
\hline Product and setup & $\begin{array}{l}\text { Wet mass consistency } \\
{[\mathrm{N} \mathrm{m} / \mathrm{kg}]}\end{array}$ & $\begin{array}{l}\text { Granule strength } \\
\text { [MPa] }\end{array}$ \\
\hline Mi-Pro Lactose/MCC 7/3 & $3.11 \pm 0.71$ & $18.1 \pm 3.29$ \\
Diosna $(\mathrm{Fr}=\mathrm{ct}$ ) Lactose/MCC 7/3 & $3.03 \pm 0.72$ & $10.1 \pm 2.03$ \\
Diosna $(\omega=\mathrm{ct})$ Lactose/MCC $7 / 3$ & $2.82 \pm 0.73$ & $8.9 \pm 2.04$ \\
Mi-Pro Lactose/MCC 3/7 & $4.15 \pm 0.72$ & $26.46 .1 \pm 5.5$ \\
Diosna $(\mathrm{Fr}=\mathrm{ct})$ Lactose/MCC 3/7 & $3.81 \pm 0.79$ & $24.48 \pm 6.02$ \\
Diosna $(\omega=\mathrm{ct})$ Lactose/MCC 3/7 & $3.56 \pm 0.86$ & $21.44 \pm 4.15$ \\
\hline
\end{tabular}

could also be a function of MCC shrinkage upon drying which was also observed by Kristensen et al. [1].

\subsection{Granule strength}

Granule strength has been assessed by two methods in an effort to describe both wet and dry granules: wet mass consistency measurements, for the wet end granules and uniaxial compression tests, for the end dry granules. Similar values of wet mass consistency have been obtained for the two formulations with only pure MCC showing a noticeably larger wet mass consistency (Table 2 ). In terms of granule strength we observe an increase in the mean dry granule strength with increasing MCC content. The opposite is observed when looking at the displacement (Fig. 14). The formulation Lactose/MCC 7/3 shows larger displacement values up to the fracture point indicating an increased plasticity of the granules.

\subsection{Effect of mixer design}

Effect of mixer design was evaluated by applying constant impeller tip speed (Eq. (4)) and constant Froude number (Eq. (5)). These rules gave two corresponding impeller speeds on the Diosna: $460 \mathrm{rpm}$ (impeller tip speed) and $605 \mathrm{rpm}$ (Froude). The same fill ratios, granulation time, optimum $\mathrm{L} / \mathrm{S}$ ratios have been kept while using a manually controlled drop by drop pump on both high shear mixers. The chopper on the Diosna has not been operated while the chopper on the Mi-Pro was turning at $3000 \mathrm{rpm}$.

$\omega=\frac{N}{60} \cdot \pi \cdot D$

$F r=\frac{N^{2} \cdot D}{g}$

where $N$ is the impeller speed, $D$ the impeller diameter and $g$ the gravitational acceleration constant.

We keep an impeller tip speed of $5.85 \mathrm{~m} / \mathrm{s}$ while passing to the Diosna while the constant Froude number gives an impeller tip speed of $7.72 \mathrm{~m} / \mathrm{s}$.

Mean granule size analysis (Fig. 15) shows that both scale-up rules yield lower mean granule sizes on the Diosna than on the Mi-Pro. In this figure ITS is indicative of the scale-up rule used meaning impeller tip speed while Fr is indicative of the Froude number being used as a scale-up rule. Also the granule size distributions present a more pronounced bi-modality showing a larger proportion of fine particles on the Diosna. This could be related to the differences in the binder addition method. On the Mi-Pro the binder flow is directed towards the chopper in order to ensure better binder distribution. On the Diosna binder addition is done with a capillary twice as large and binder flow cannot be directed towards the chopper. The difference in granule size distribution can be explained by the formation of lumps (granules with sizes above $5 \mathrm{~mm}$ ) representing about $10 \%$ of the mass despite the high impeller speeds on the Diosna. On the Mi-Pro the formation of lumps has not been observed. Constant impeller tip speed yields a closer result in terms of granule size (Table 3). It shears and impacts less the wet granules than the constant Froude number. It should also be noted that while for the Lactose/MCC 3/7 formulation scale-up rules produce roughly the same results with differences in the final mean granule size inferior to $8 \%$, the lactose formulation is more sensitive to changes in impeller speed. At a constant impeller tip speed we observe a decrease in the mean granule size of $9.2 \%$ and at a constant Froude number of $17.7 \%$. Usual variations in granule size between batches in the same operating conditions have been between 3 and $10 \%$. The difference in mean granule size is probably not a function of increased breakage occurring in the Diosna but rather a result of the formation of lumps giving an inhomogeneous binder distribution. From a morphological point of view, SEM observations presented in Fig. 16 have not allowed us to identify differences in growth mechanisms between the two scales.

Table 4 presents the recorded wet mass consistency values and dry granule strength measurements for the experiments on both scales. We observed a higher consistency for the Lactose/MCC 3/7 formulation than the Lactose/MCC 7/3 formulation indifferent of the scale used with wet mass consistency decreasing on the large scale. For the large scale (Diosna) we observed that increasing impeller speed also increases wet mass consistency. Although values remain fairly close it is not unreasonable to imagine that increased impeller speed squeezes more binder to the granule surface. Dry granule strength is found to decrease on the Diosna for both formulations regardless of scale-up rule used. However, increasing impeller speed on the Diosna increases dry granule strength. As a consequence we can say that using the Froude number as a scale-up rule gives a better agreement in terms of mean dry granule strength. For the case of the Lactose/MCC $7 / 3$ formulation mean granule size is halved upon scale-up. We attributed this phenomenon to a preferential agglomeration of the MCC particles in the lumps formed on the Diosna. Because of this, the granules poor in MCC content show lower mean granule strength.

\section{Conclusion}

Torque curves allow following agglomeration mechanisms at the macro scale which were confirmed by scanning microscope analysis and granule growth kinetics. The water sorption isotherms have shown a greater affinity of MCC for water vapor than lactose and this behavior is recognized in the granulation mechanism of the two formulations. They also seem to indicate that water becomes loosely bound at particle surface quicker for lactose than for MCC. This could explain the shorter wetting periods for increasing lactose content as well as the accelerated growth associated. The proposed growth mechanism seemed to be independent of the scale used for investigation.

The Lactose/MCC 3/7 formulation showed both higher consistencies and higher dry granule strength on both scales. The difference is found to be greater in terms of dry granule strength than in terms of wet mass consistency. For the Lactose/MCC $7 / 3$ formulation dry granules are more deformable, granules supporting greater irreversible deformation until fracture appears.

The effect of mixer design studied by scaling up from the 1.9 Mi-Pro to the 6 L Diosna high shear mixer showed better agreement in terms of mean granule size when using the impeller tip speed as a scale-up rule. However this agreement seems of a lesser importance when analyzing granule size distributions. Both scale-up rules yield a higher percentage of lumps and fine particles presenting significantly different granule size distributions from the ones obtained on the smaller scale. This could be explained by differences in binder addition and dispersion systems between the scales. Both wet mass consistency and dry granule strength for the studied formulations decreased upon scale-up with a more accentuated difference in terms of dry granule strength. 


\section{References}

[1] J. Kristensen, T. Schæfer, P. Kleinebudde, Direct pelletization in a rotary processor controlled by torque measurements. II: Effects of changes in the content of microcrystalline cellulose, AAPS Pharmsci 2 (3) (2000) article 24

[2] J. Kristensen, P. Holm, A. Jaegerskou, T. Schæfer, Granulation in high speed mixers Part 4: effect of liquid saturation on the agglomeration, Pharm. Ind. 46 (1984) 763-767.

[3] C. Vecchio, G. Bruni, A. Gazzaniga, Preparation of indobufen pellets by using centrifugal rotary fluidized bed equipment without starting seeds, Drug Dev. Ind. Pharm. 20 (12) (1994) 1943-1956.

[4] P. Holm, M. Bonde, T. Wigmore, Pelletization by granulation in a roto-processor RP-2-Part 1: effect of process and product variables on granule growth, Pharm. Technol. 8 (1996) 22-36.

[5] H.L. Leuenberger, M. Puchkov, E. Krausbacher, G. Betz, Manufacturing pharmaceutical granules: is the granulation end-point a myth, 3rd Int. Granulation Workshop Sheffield, 2007.

[6] G. Betz, P.J. Burgin, H.L. Leuenberger, Power consumption profile analysis and tensile strength measurements during moist agglomeration, Int. J. Pharm. 252 (2003) 11-25

[7] M.B. Mackaplow, L.A. Rosen, J.N. Michaels, Effect of primary particle size on granule growth and endpoint determination in high-shear wet granulation, Powder Technol. 108 (2000) 32-45.

[8] R.L. Carr, Evaluating flow properties of solids, Chem. Eng. 18 (1965) 163-168.

[9] J. Schwedes, Testers for measuring flow properties of particulate solids, Powder Handling Process. 12 (4) (2000) 337-354.

[10] R.C. Rowe, Characterization of wet powder masses using a mixer torque rheometer. 4. Effect of blade orientation, Int. J. Pharm. 133 (1996) 133-138.
[11] P. Luukkonen, T. Schæfer, L. Hellen, A.M. Juppo, J. Yliruusi, Rheological characterization of microcrystalline cellulose and silicified microcrystalline cellulose wet masses using a mixer torque rheometer, Int. J. Pharm. 188 (1999) 181-192.

[12] P. Kleinebudde, The crystallite-gel-model for microcrystalline cellulose in wet granulation, extrusion and spheronization, Pharm. Res. 14 (1997) 804-809.

[13] J.S. Ramaker, Fundamentals of high shear pelletisation process - PhD thesis, Rijksuniversiteit Groningen (2001).

[14] M.J. Adams, M.A. Mullier, J.P.K. Seville, Agglomerate strength measurement using a uniaxial confined compression test, Powder Technol. 78 (1994) 5-13.

[15] F. Rouquerol, J. Rouquerol, S. King, Adsorption by Powders and Porous Solids: Principles, Methodology and Applications, Academic Press, 1999.

[16] T.M. Chitu, D. Oulahna, M. Hemati, Wet granulation in a laboratory scale high shear mixer: effect of chopper presence, design and impeller speed, 9th Int. Symposium on Agglomeration Sheffield, 2009.

[17] M. Benali, Prédiction des interactions substrat/liant lors de la granulation: Etude expérimentale dans un mélangeur granulateur à fort taux de cisaillementApproches thermodynamiques par simulation moléculaire, PhD thesis, INP Toulouse (2006).

[18] A. Goldszal, J. Bousquet, Wet agglomeration of powders: from physics toward process optimization, Powder Technol. 117 (2001) 221-231.

[19] P. Kleinebudde, Shrinking and swelling properties of pellets containing microcrystalline cellulose (MCC) and low substituted hydroxypropylcellulose (L-HPC). I. Shrinking properties, Int. J. Pharm. 104 (1994) 209-219.

[20] M. Kuentz, $H$. Leuenberger, A new theoretical approach to tablet strength of binary mixture consisting of a well and a poorly compactable substance, Eur. J. Pharm. Biopharm. 49 (2000) 151-159. 